\title{
The Semantic Architecture for Chinese Cultural Celebrities' Manuscript Library
}

\author{
Wei Liu \\ Shanghai Library, No.1555, Huai Hai Zhong Lu, Shanghai 200031, China \\ wliu@libnet.sh.cn
}

\begin{abstract}
Semantic architecture is crucial for a digital library application especially in a distributed system environment. It provides various approaches to overcome semantic interoperability problems and usually consists of metadata solution with open system architecture. The design of the digital library system for the China Cultural Celebrities' Manuscripts Library (CCCML), which is a branch of Shanghai Library, has taken into account a lot of the main aspects from the requirement of semantics, including the metadata profiles, encoding consistence, authority control, ontology functioning, semantic integration, etc. We argue that it is very important to establish an articulated layered semantic architecture for digital libraries in the semantic web environment. And it becomes more and more clear that the semantic services can be settled with the Semantic Web Services technologies, which is supported by and consisted of a wide range of standards and protocols. And a lot of mainstream interoperability architecture, such as OAI, OpenURL etc., can be conformed or implemented by Semantic Web Services. This paper gives some major considerations and overviews on the design of semantic architecture for CCCML, which shows a lot of similarity in typical digital library systems.
\end{abstract}

\section{Introduction}

Metadata is usually defined as data about data, and semantics is the meaning of meaning. When computing involving with semantics, metadata is becoming more and more obvious important to be the semantic building blocks of all kinds of information systems such as digital libraries. But metadata itself, without some sort of mechanism, including term selection, profile composition, encoding formalization, ontology annotation, vocabulary mapping, authority controlling and service allocation etc., will not realize its full potential to implement semantics. In this paper, we call such a mechanism the "semantic architecture".

The most significant and difficult requirement for digital libraries (usually considered as distributed information systems on the web to bring together collections and services) is to achieve a high level of "semantic interoperability". We can never expect that digital library applications will be developed with a uniformed data model or conformed to just a few metadata sets, such as Dublin Core, EAD, VRA Core etc. There will be emerging numerous domain specific metadata sets continuously, while 
at the same time the sharing of metadata standards becomes widely accepted and popular. We have to find some ways to deal with the heterogeneous problem and to integrate the diversity information systems of a digital library into one consolidated view.

An articulate layered semantic architecture will help us to achieve such a goal. With the design and development of the digital library system for Chinese Cultural Celebrities' Manuscript Library (CCCML), we propose and implement a semantic architecture to accomplish a high level of interoperability and to reserve a good scalability, extensibility and integratibility for the system. But in this paper we are not focusing on the technical details of the implementation and arithmetic of the developing, such as schema matching, ontology mapping etc. The remainder of this paper is structured as follows. The next section introduces the semantic requirements of the CCCML digital library system. Section 3 reviews some related works in this area. Section 4 describes a semantic architecture which is the main contribution of the paper, following with the Section 5: the future consideration under the emerging technology of Semantic Web Services.

\section{CCCML Application Requirements}

Unlike a traditional library, CCCML has a collection with tens of thousands various kinds of documents and physical objects, including manuscripts, letters, diaries, photographs, books with signature and remarks, notebooks, account books, paintings, calligraphies, seal cuttings, badges, diplomas, print materials as well as audio visual materials. A large portion of the collections is expected to be digitized in the near future to provide better preservation and services. Items in the collections are all connected with (made by or related to) "Chinese cultural celebrities" and can be referred or linked to other related applications like OPAC system, union cataloguing system and inventory systems of special libraries and museums across the country.

The CCCML digital library can be roughly considered into three sub-systems: the Digitalization System (DS), the Metadata Management System (MMS) and the Digital Object Repository System (DORS), in which all consist of software modules or components and interconnected with each other (see Fig. 1). We take the MMS as the key system to be designed and accomplished at the first phase before the end of 2004. It provides the base data and information model and shapes the overall architecture of the digital library.

The goal of the CCCML Digital Library System is to provide a digital repository with preservation and retrieval services for the resources of Chinese Cultural Celebrities' collections in the Shanghai library. The application should make the full use of IT infrastructure and digital library architecture which have been developed and maintained since 1999, and integrated into the whole digital resources and services framework within Shanghai Library. It is by no means another stand alone autonomous system for a series of special collections. So it should be designed to be component based and loose coupled, even to share the same software environment and server capabilities with other applications. 


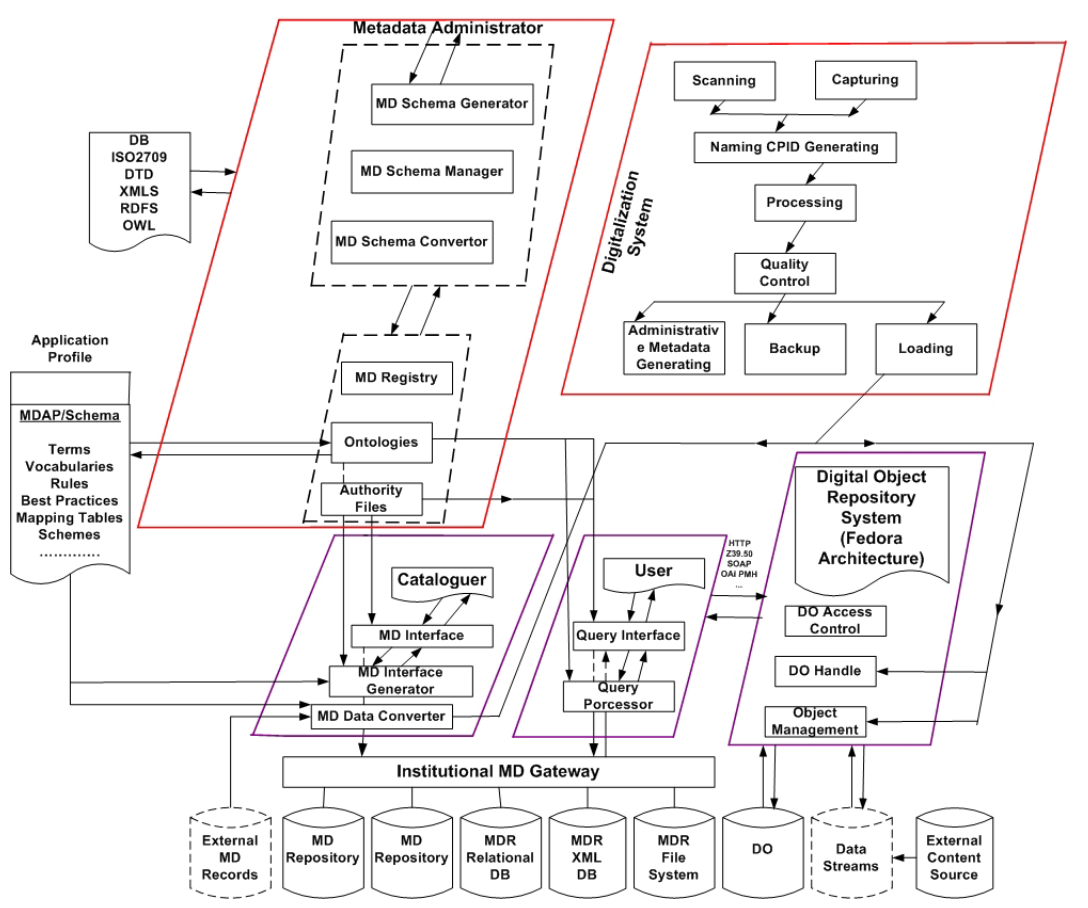

Fig. 1. The system architecture of CCCML

We have encountered several special requirements and difficulties regarding the semantic discovery in construction of CCCML digital library system:

1. The Variety Types of Resources in CCCML collections are always with different but overlapping properties need to discover, at the same time all the metadata terms should be "standard" and conforms to Metadata Guidelines of the Shanghai Library, which contains a "core set" of metadata elements derived from DC-Lib application profile, with a set of encoding rules and best practices for metadata manipulating. Metadata Application Profile (MAP) provides a practical approach to fulfill domain specific description needs while remains compatibility with major metadata standards. But the implementation of MAP is still in its early stage with ambiguous in a few aspects like qualification and keeping encoding consistency. And MAP encoded with XML Schema cannot represent semantic restrictions and formalize all constrains required by the system.

2. Each type of resource has its own metadata profile expressed with XML Schema. So the system should support multi-schema management, including input, load, open, edit, parse, error detect, and convert between different types of schemas: DTD, XML Schema, RDF Schema etc. The most important function is to generate type and context aware interfaces for metadata instance manipulation (input, edit, convert, output, and storage) according to different types and properties of resources. 
3. Complicated Relations between agents (person, institution), objects, and their properties should be described explicitly and precisely. For the Cultural Celebrities always have a lot of social relations and changing roles and attributes during their life long time. These need to be documented in order to establish all kinds of relations between objects related to them in collections. Ontology could be an ideal and powerful tool to map these complex relations and provide a comprehensive view for modeling the system.

4. Authority Control provides consistency and permanence of a name or a concept. In traditional library system, it creates a link between bibliographic records and authority file, and forms the underlying structure of the catalog. Similar to the use of ontology, authority control with the name, affiliation, event, and subject of Culture Celebrity can aggregate the related records without precision lost, and provide multiple dimensions to navigate the repository.

5. With the expansion of digital collections in the Shanghai Library, no matter the resources acquired from various dealers or digitized by its own staffs, as well as in the shape of physical media or access to an outside website or virtual portal, the integration of resources and services will always be the strategic task in the highest priority. The service of CCCML collections is expected to be integrated into Digital Library System in Shanghai Library, which is still under developing to become a multilayer loose coupled opening architecture, as a result of the realization of FEDORA system. So the data model should be quite flexible and can support a lot of metadata standards with METS $^{1}$ as a standard schema container. The use of web service exposure layer in FEDORA adopted a lot of communication protocols such us HTTP, SOAP, OAI, Z39.50 etc., will bring the CCCML digital library a wide range of interoperability and adaptability.

\section{Related Work}

Semantic interoperability is a major challenge in resources and services integration. Semantic heterogeneity comes from the mismatch in meanings with different tags represented by different terms from different vocabularies, when expressed with different schemas conformed to different guidelines. There are various kinds of conflicts that cause semantic inconsistencies with naming, data structure, attributes, granularity, types of values, etc. We think an integrity semantic architecture can help a lot to facilitate the semantic interoperability between systems.

This paper was inspired by FEDORA ${ }^{2}$, HARMONY $^{3}$ and ARIADNE ${ }^{4}$ projects, as well as some researches on enterprise data integration [11][12]. Semantic Web as a

\footnotetext{
1 See: http://www.loc.gov/standards/mets/

2 See: http://www.fedora.info/

3 http://www.ilrt.bris.ac.uk/discovery/harmony/

4 http://www.ariadne-eu.org/
} 
significant movement about Web technologies, which aims to move from syntactic interoperability to semantic interoperability and relies on machine interpretable semantic descriptions, is also a technical resource for the designing of Semantic Architecture of CCCML digital library.

Chen's paper [1] reviews two results of semantic research from the early digital library projects: feasible scalable semantics and semantic indexes of large collections. They all deal with the retrieval effectiveness of massive information instead of description and architecture aspect of the interoperability solution for heterogeneous repositories. Norm Friesen [3] analyzed the meaning of semantic interoperability and metadata approach to achieve it in detail, but with a somehow pessimistic conclusion- "The goal of increased interoperability ... will clearly not be achieved through further formalization and abstraction". ABC ontology [4][5] from Harmony project proposed by Carl Lagoze, Jane Hunter etc. derived from FRBR ${ }^{5}$ can model the complexity of relations between resources and properties. It is a good abstract model for resource integration, but for the lack of application specifications, it does not provide an articulate semantic architecture and can be implemented in different levels with different approaches. Paper [8] by Jérôme Euzenat roughly layered interoperability into five levels: encoding, lexical, syntactic, semantic, and semiotic based on a classification of possible requirements, from which each level can not been achieved without the completion of the previous one. The discussion is based on the purpose of implementation of a totally machine executable semantic representation and transformation on Semantic Web. These researches shed light on the approach of establishing a semantic architecture for CCCML digital library.

\section{The Semantic Architecture}

\subsection{The Purpose}

Semantic architecture brings structure to the content of a digital library. The structure can expose some interfaces to outside world accessed by people as well as mediator agents. The design of semantic architecture is to give a practical approach under the consensus of semantic interoperability within and between communities.

We see the main purpose to establish a semantic architecture is to formalize the semantic description of digital resources, for the better serving of resource and service discovery and exposing adequate interfaces for the integration of digital resources, and finally to achieve high level interoperability between digital libraries.

\subsection{The Approach}

The specification of Metadata Application Profile (MAP) provides the foundation of a semantic architecture for digital libraries. MAP is defined as a kind of metadata schema which consists of data elements drawn from one or more namespaces,

\footnotetext{
${ }^{5}$ FRBR: Functional Requirements for Bibliographic Records. see: http://www.ifla.org/II/s13/br/frbr.pdf
} 
combined together by implementers, and optimized for a particular local application [17]. It becomes a standard approach with methodologies and procedures to reuse metadata terms from various metadata standards authorities, share the semantics and structures all in once without the burden of setting up one's own metadata registry. One example of MAP is a CEN standard: CWA14855- "Dublin Core Application Profile guidelines", which is a declaration specifying which metadata terms to use and how these terms have been customized or adapted to a particular application. But it stopped in terminology level which can help to share a common data model underlying the applications but not information model which specifies complex relations among resources and properties during its life cycle.

The use of controlled vocabularies (thesauri), authority files and ontologies are practical means of system level to achieve consistency and integrity within and between digital libraries. To get the better flexibility and extensibility, especially in large institutions or enterprises with a number of various kinds of information resources and applications, the metadata registries which collect and maintain data dictionaries, metadata elements, schemas and vocabularies are the sources and repositories of formal semantics. They are the key mechanism to the semantic architecture, especially when the registries can provide web services for software agents by the request of digital library applications.

\subsection{The Implementation}

The semantic architecture for CCCML consists of schemas in data model level (which consists of the formal definition and restrictions of "core" elements, extended elements, metadata profiles, schema encoding rules) and information model level (which consists of relations between elements, ontologies, procedures and methodologies and Institutional registry for local qualified terms, schemas and namespaces), which serves for consistent description and discovery of semantics of the resources in CCCML. The architecture takes the form of a collection of schemas, tools and documentations which support semantics manipulating needs within the life cycle of the resources. The following paragraphs introduce the semantic architecture of CCCML system in a sequence of workflow:

\section{Resource Analysis and Definition}

The resources in the CCCML collections are defined from a practical point of view, from which the system can never anticipate what a set of properties of next object will be. We predefined twelve categories of resources with fixed metadata set and encoding schema in a form of Metadata Application Profile. But the system can accept multiple number and any kinds of MAP at the same time in the form of DTD, XML Schema or RDF Schema. The only necessity is the category of a resource should be defined explicitly with a set of properties (metadata elements from multiple namespaces with definitions), guidelines for cataloguing and encoding.

2. Metadata Set Definition (Core and Extended)

Shanghai Library had issued a specification with a "Core" set of metadata elements and encoding guidelines for the interoperation of all its digital library applications. The specification derived elements from DC-Lib application profile and takes a 
reference to "the IFLA Guidance on the Structure, Content, and Application of Metadata Records for Digital Resources and Collections"6. As a digital library application of Shanghai Library, the CCCML system takes the "Core" as its mandatory set of elements. But this does not mean every element should be in use with the resource of CCCML. The element in the "Core" only becomes mandatory when it is needed.

At the same time each type of resources in CCCML 'borrows' some elements from other metadata standards like MODS, VRA Core etc., and proposes its own elements, as its the domain specific MAP. So a local metadata registry should be established to maintain terms in a local namespace for the proposed elements as well as for those terms from other metadata standards without namespaces.

It is not recommended to invent elements or terms for any resource. But the content owners and users of CCCML want to discover the properties of the resources exhaustively. So we developed a rigorous procedure for approving the proposed terms.

\section{Encoding and Mapping Rules}

The Schema Suite is a stand alone utility to manipulate (open, load, input, parse, edit, save, delete, convert, output etc.) metadata schemas and generate web interfaces for metadata cataloguing as well as help to generate the query interface. It is designed to support DTD, XML Schema and RDF Schema according to the rules of encoding from time to time. All empty schemas (without instances) can be kept and managed with the tool.

Basically the tool is fed with an original schema of the "Core" set. But it supports aliases for core elements so that it can be user-friendly to the domain expert for inputting and retrieving to the resources. It can support to accept records with ISO2709 format and transform it to any form of a MAP according to a mapping table.

\section{Guidelines and Best Practices}

For the limitation on the capabilities of different formalization language like XML Schema or RDF Schema, not all of the restrictions and constrains can be expressed and encoded with them. Some of the functions have to accomplish during implementation.

So the metadata element set and its encoding is not enough to carry the semantics of an information model. It must assist with restrictions, constrains, rules, guidelines etc. That's why the semantic architecture has documentation for people readable instead of machine readable. All these documents would better be kept and maintained in a mechanism of registry system so as to provide open access by people or agents. What's more it can be extended to construct web service to provide semantic support services (discussed below).

5. Metadata Registry, Ontologies and Authority Files

Registries are essential to the scalability of a digital library, for it provides a mechanism in the distributed environment to get the semantic architecture reusable, sharable, integrity and consistence. Local registry is a kind of "have-to" facility for institutions and enterprises as the scale of application becomes bigger and bigger and

${ }^{6}$ See: http://www.ifla.org/VII/s13/guide/metaguide03.pdf 
eventually get out of control. Registry can be considered as data dictionary for local systems. But the metadata registry should synchronize with open registries distributed on the internet. And it's better to open itself to serve as a member of the metadata registry cluster.

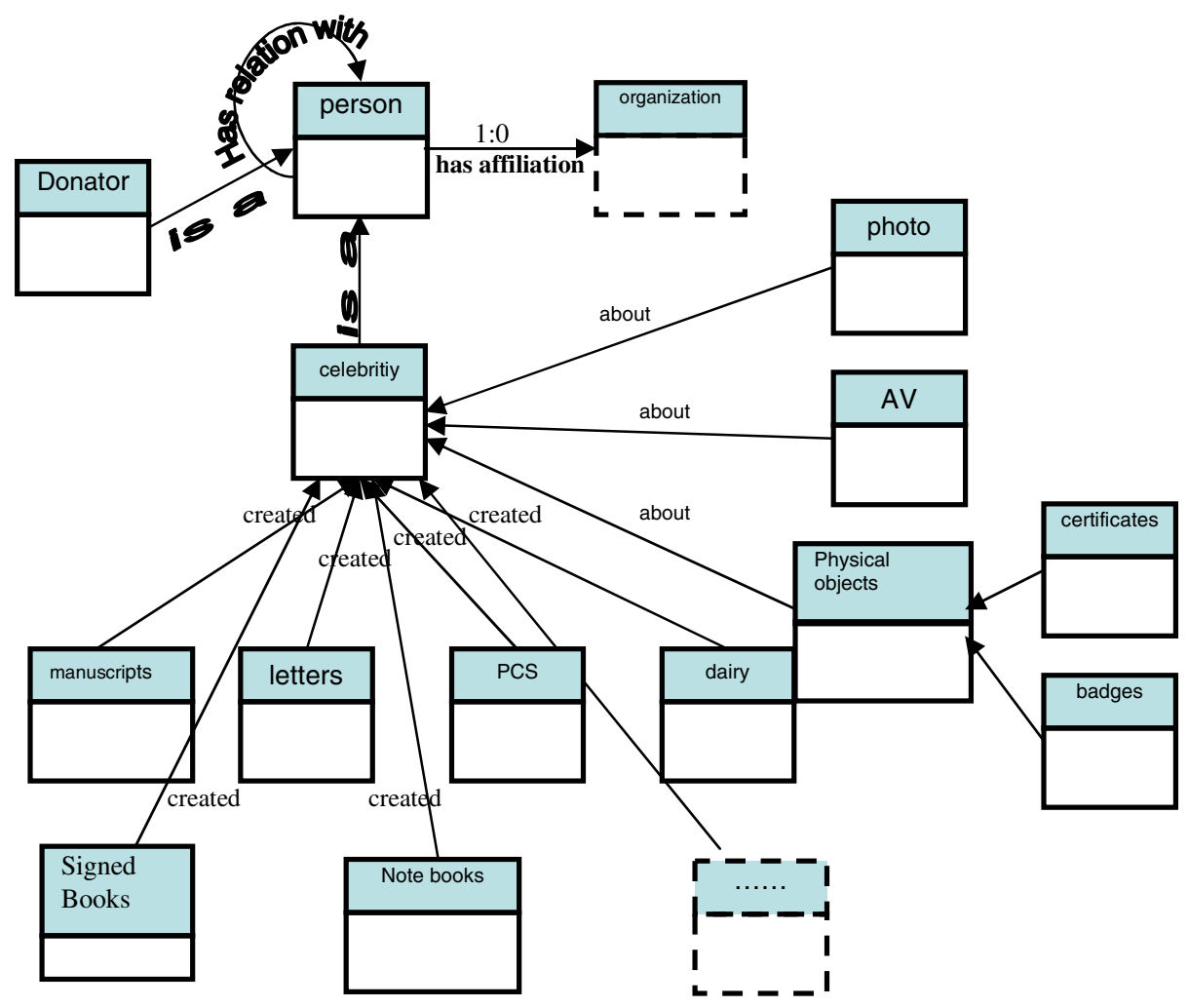

Fig. 2. The ontology of CCCML resources

Ontology brings the semantic integrity of a digital library. The formalization of an information model which consist of metadata profile and relations between objects and properties within a digital library can be considered as a ontology. Fig. 2 illustrated the ontology of CCCML in brief. Some resources and properties in the ontology should be controlled with authority files, such as the "person" in Fig.2, and some subject properties can be controlled with encoding scheme.

\section{Future work}

There are three kinds of implementation models to accomplish the semantic architecture for consistency and interoperability among applications: 
a) Simple model: schema/mapping;

b) Formal model: registry and agent mediator; and

c) Full functional model: metadata services by means of semantic web services which depends on a series of standards and protocols to be settled down.

We just proposed an implementation in level b) mentioned above. The registry function we plan to realize illustrated in Fig. 3. For the second phase of the project, we plan to accomplish FEDORA Architecture to set up digital objects repository. And we expect that the system will eventually support multi-interoperable linkage with protocols such as OpenURL, OAI, and Z39.50.

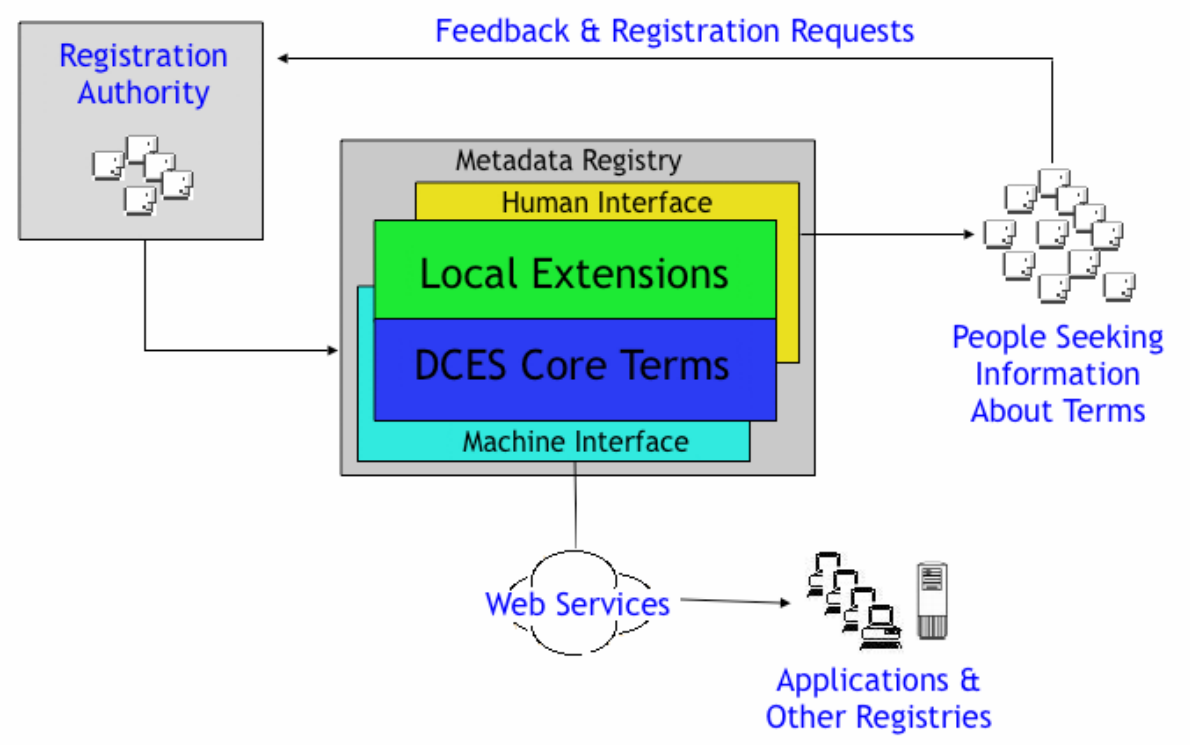

Fig. 3. Single metadata registry overview ${ }^{7}$

\section{References}

1. 1.Chen, H.: Semantic Research for Digital Libraries. D-Lib Magazine. Oct 1999. http://www.dlib.org/dlib/october99/chen/10chen.html ()

2. Schreiber Z.: Semantic Information Architecture: Creating Value by Understanding Data published in DMReview.com October 1, 2003 http://www.dmreview.com/articlesub.cfm? articleId $=7438()$

3. Friesen, N,: Semantic Interoperability and Communities of Practice. February 5, 2002 http://www.cancore.ca/documents/semantic.html ()

7 From Harry Wagner's presentation on the "Open Forum for eBusiness and Metadata Technology Standardization” Xi'an, China, May 2004. 
4. Hunter, J., Lagoze, C.: Combining RDF and XML Schemas to Enhance Interoperability Between Metadata Application Profiles. http://www.cs.cornell.edu/lagoze/papers/ HunterLagozeWWW10.pdf ()

5. Doerr M., Hunter J., Lagoze C.: Towards a Core Ontology for Information Integration. Journal of Digital Information, Volume 4 Issue 1. (Article No. 169, 2003-04-09) http://jodi.ecs.soton.ac.uk/Articles/v04/i01/Doerr/

6. Amit Sheth, Vipul Kashyap , and Tarcisio Lima: Semantic Information Brokering: How Can a Multi-Agent Approach Help? http://cgsb2.nlm.nih.gov/ kashyap/publications/cia.doc

7. Jehad Najjar, Erik Duval, Stefaan Ternier, Filip Neven: TOWARDS INTEROPERABLE LEARNING OBJECT REPOSITORIES: THE ARIADNE EXPERIENCE. Proc. IADIS Int'1 Conf. on WWW/Internet 2003, Vol. I, P. 219-226. ISBN 972-98947-1-X

8. Jérôme Euzenat: Towards a principled approach to semantic interoperability. http://ceurws.org/Vol-47/euzenat.pdf

9. Marco Schorlemmer, Yannis Kalfoglou: Using Information-Flow Theory to Enable Semantic Interoperability. Informatics Research Report EDI-INF-RR-0161. March 2003

10. Deborah L. McGuinness. "Ontologies Come of Age". In Dieter Fensel, J im Hendler, Henry Lieberman, and Wolfgang Wahlster, editors. Spinning the Semantic Web: Bringing the World Wide Web to Its Full Potential. MIT Press, 2002.

11. Michael Breu, Ying Ding: Modelling the World: Databases and Ontologies.

12. ZVI SCHREIBER: SEMANTIC INFORMATION MANAGEMENT (SIM): SOLVING THE ENTERPRISE DATA PROBLEM BY MANAGING DATA BASED ON ITS BUSINESS MEANING. 2003(V2).

13. Maria Inês Cordeiro, Aida Slavic: Data Models for Knowledge Organization Tools: Evolution and Perspectives. Challenges in knowledge representation and organization for the 21st century: integration of knowledge across boundaries: proceedings of the the Seventh International ISKO Conference, 10-13 July 2002, Granada, Spain). Eds. María J. López-Huertas. Ergon Verlag, 2002. (Advances in Knowledge Organization; Vol 8). pp. 127-134. ISBN 3-89913-247-5

14. Albert Benschop: The future of the semantic web. http://www2.fmg.uva.nl/sociosite/ websoc/semantic.html.

15. Sheila A. McIlraith, Tran Cao Son, and Honglei Zeng: Semantic Web Services. IEEE INTELLIGENT SYSTEMS MARCH/APRIL 2001 pp46-53.

16. Abhijit Patil, Swapna Oundhakar, Amit Sheth: Semantic Annotation of Web Services (SAWS). http://lsdis.cs.uga.edu/ abhi/SAWS-TR.htm.

17. Thomas Baker, Makx Dekkers, Rachel Heery, Manjula Patel, and Gauri Salokhe, "What Terms Does Your Metadata Use? Application Profiles as Machine-Understandable Narratives". Journal of Digital Information, Volume 2 Issue 2 (November 2001)

18. (Acknowledgement to my colleague Leon Zhao for his inspired discussion with me occasionally and to Miss Lu Ying for drawing the Fig. 1 for me) 